\title{
Toxicokinetics of Riddelliine, a Carcinogenic Pyrrolizidine Alkaloid, and Metabolites in Rats and Mice
}

\author{
Lee Williams,* Ming W. Chou,* Jian Yan,* John F. Young,* Po C. Chan, $\dagger$ and Daniel R. Doerge*,1 \\ *National Center for Toxicological Research, Jefferson, Arkansas 72079; and $\dagger$ National Institute for Environmental Health Sciences, \\ Research Triangle Park, North Carolina 27709
}

Received December 12, 2001; accepted April 30, 2002

Toxicokinetics of Riddelliine, a C arcinogenic Pyrrolizidine Alkaloid, and Metabolites in Rats and Mice. Williams, L., Chou, M. W., Y an, J., Young, J. F., Chan, P. C., and Doerge, D. R. (2002). Toxicol. Appl. Pharmacol. 182, 98-104.

Riddelliine is a representative pyrrolizidine alkaloid, a class of naturally occurring toxic phytochemicals present in plant species worldwide. Human exposure to pyrrolizidine alkaloids occurs through consumption of herbal dietary supplements, including comfrey, and through contaminated livestock products (e.g., milk). A recently completed 2-year bioassay of riddelliine carcinogenicity showed that male and female rats and male mice, but not female mice, developed liver tumors. The toxicokinetics of riddelliine and two metabolites, the $N$-oxide and retronecine, were determined in serum following an oral gavage dose in male and female rats and mice using a validated liquid chromatographyelectrospray mass spectrometric method. The results are consistent with extensive metabolism of riddelliine and its more polar metabolites prior to excretion. It is concluded that factors other than toxicokinetics are responsible for the observed species/sex specificity of gross toxicity or liver tumor induction in rats and mice.

Key Words: riddelliine; pyrrolizidine alkaloid; mass spectrometry; toxicokinetics.

Riddelliine (see Fig. 1 for structures) belongs to a class of naturally occurring compounds known as pyrrolizidine alkaloids that are contained in many plant species around the world (International Agency for Research on Cancer, 1976). Riddelliine has been isolated from plants of the genera Crotalaria, Amsinckia, and Senecio, which are present in the rangelands in the western United States. The high toxicity of these compounds has caused great loss of free-ranging livestock due to liver and pulmonary lesions (Mattocks, 1986). More disturbing is the possible contamination of human food stuffs such as meat, milk, honey, and herbs (Chan et al., 1994). Commercially available comfrey dietary supplements and the herbal tea "gordolobo yerba," popular in the American Southwest, also

${ }^{1}$ To whom correspondence should be addressed. Fax: (870) 543-7720; E-mail: ddoerge@nctr.fda.gov. contain pyrrolizidine alkaloids (Hirono et al., 1976; Huxtable, 1980; Betz et al., 1994; Roeder, 1995). Based on these possible hazards to human health, the U.S. Food and Drug Administration nominated riddelliine for genotoxicity and carcinogenicity testing conducted by the National Toxicology Program (NTP). The NTP 2-year study showed that riddelliine induced liver hemangiosarcomas in both male and female rats and male mice, hepatocellular adenomas and carcinomas in male and female rats, and lung alveolar adenomas in female mice (National Toxicology Program, 2001). The genotoxic effects of riddelliine, and presumably other pyrrolizidine alkaloids, are thought to arise through metabolic activation of the parent compounds by hepatic cytochromes P450 (Yang et al., 2001b). Dehydroretronecine, the reactive metabolite of riddelliine, forms several DNA adducts that have been quantified using ${ }^{32} \mathrm{P}$-postlabeling analysis (Yang et al., 2001a). Scheme 1 shows the proposed metabolic reactions leading from riddelliine to DNA adducts.

A critical role of oxidative metabolism in the carcinogenic bioactivation, and conversely the hydrolytic detoxication, of pyrrolizidine alkaloids was supported by many previous investigations (cf. Yang et al., 2001b). In addition, clear sex and species differences in the toxicity of riddelliine to rodents were reported by Chan et al. (1994). For these reasons, it was important to compare the metabolism of riddelliine in both sexes of rats and mice and to determine the toxicokinetics of riddelliine and metabolites in both species to understand better the biological mechanisms affecting the tumorigenic process. These studies required development and validation of an offline solid phase extraction procedure for sample purification coupled with a sensitive liquid chromatography-electrospray mass spectrometric (LC/ES-MS) method for concurrently quantifying riddelliine and its two major metabolites from serum. This approach facilitated a more detailed investigation of the metabolism and disposition of a pyrrolizidine alkaloid than that possible using a radiolabeled substrate.

\section{MATERIALS AND METHODS}

Reagents. The riddelliine used was the same as that previously characterized (Chan et al., 1994) and a current LC/MS analysis indicated no deterio- 
<smiles>CC=C1CC(C)[C@@](O)(CO)C(=O)OCC2=CCN3CC[C@H](OC1=O)C23</smiles>

Riddelliine<smiles>OCC1=CCN2CC[C@H](O)[C@H]12</smiles>

Retronecine<smiles>C=C1C/C(=C/C)C(=O)O[C@H]2CCN3CC=C(COC(=O)[C@](O)(CO)C1=O)[C@H]23</smiles>

Riddelliine $\mathrm{N}$-oxide<smiles>OCc1ccn2c1[C@@H](O)CC2</smiles>

Dehyroretronecine<smiles>C[C@@H]1C(=O)O[C@H]2CCN3CC=C(CO[C@H](C)[C@@](C)(O)[C@@H]1O)C23</smiles>

Monocrotaline

FIG. 1. Structures for pyrrolizidine alkaloids.

ration in composition or purity. Solvents were of HPLC grade, and water was purified using a MilliQ apparatus (Waters Associates, Milford, MA) and all buffer salts were analytical reagent grade provided by Sigma Chemical Co. (St. Louis, MO).

Solid phase extraction. Serum sample preparation was performed using Empore $\mathrm{C}_{18}$ solid-phase extraction (SPE) high-performance disk cartridges (7 $\mathrm{mm} \times 3 \mathrm{~mL}, 3 \mathrm{M}$ Corp., St. Paul, MN). The cartridge was activated with $2 \times$ $1 \mathrm{~mL}$ washes of methanol followed by $2 \times 1 \mathrm{~mL}$ washes with $\mathrm{H}_{2} \mathrm{O}$. Serum samples $(10-50 \mu \mathrm{L})$ were diluted with $100 \mu \mathrm{L}$ sodium phosphate buffer ( 25 $\mathrm{mM}, \mathrm{pH} 9$ ). The cartridge was then washed with $2 \times 1 \mathrm{~mL} \mathrm{H}_{2} \mathrm{O}$, and eluted with $2 \times 1 \mathrm{~mL}$ washes of methanol. The eluate was reduced to dryness using a centrifugal vacuum concentrator, reconstituted into $200 \mu \mathrm{L}$ of $20 \%$ methanol in $\mathrm{H}_{2} \mathrm{O}$ containing $4 \mathrm{ng}$ monocrotaline as an internal standard, and $50 \mu \mathrm{L}$ was injected onto the LC column.

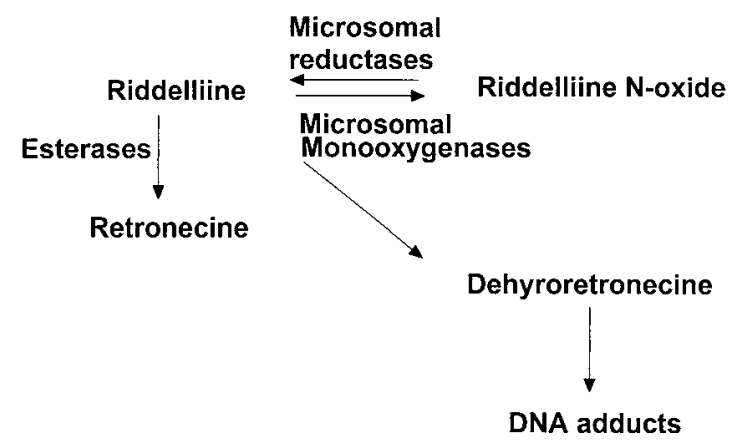

SCHEME 1. Proposed metabolic transformations of riddelliine.
Riddelliine $(\mathrm{M}+\mathrm{H})+=350$

Riddelliine $\mathrm{N}$-oxide $(\mathrm{M}+\mathrm{H})+=366$

Retronecine $(M+H)+=156$<smiles>C=CC1C=C[N+]23CCC=C2C(C)CC13</smiles><smiles>C=C1C=C[N+]2=C1C=CC[N+]2=O</smiles><smiles>CC1C=C[N+]2(O)CCC=C1C2</smiles><smiles>C=C1C=C[N+]2(O)CCC=C1C2</smiles>
-riddelliic acid, $-2 \mathrm{H}_{2} \mathrm{O}$

SCHEME 2. Proposed fragmentation reactions for riddelliine and metabolites.

Liquid chromatography. The LC separation was performed using a Dionex GP40 pump (Dionex, Sunnyvale, CA) and a Dionex AS3500 autosampler. Chromatographic separation was achieved on a Prodigy $\mathrm{C}_{18}$ column $(2 \times$ $250 \mathrm{~mm}, 5-\mu \mathrm{m}$ particle size, Phenomenex, Torrance, CA) using gradient elution (95\% of $1 \mathrm{mM}$ ammonium acetate, $\mathrm{pH} 9.0 / 5 \%$ of acetonitrile to $60 \%$ acetonitrile in $15 \mathrm{~min}$ ). The flow rate for all analyses was $0.2 \mathrm{~mL} / \mathrm{min}$, and the entire effluent was delivered to the electrospray probe.

Mass spectrometry. A Platform II single quadrupole mass spectrometer (Micromass, Manchester, UK) equipped with an electrospray interface was used with an ion source temperature of $150^{\circ} \mathrm{C}$. Positive ions were acquired in the selected ion monitoring mode (dwell time $=0.2 \mathrm{~s}$ and interchannel delay time $=0.02 \mathrm{~s}$ ). The base peak of each spectrum was monitored and, in all cases, corresponded to the respective protonated molecule $[\mathrm{M}+\mathrm{H}]^{+}$. Optimized cone-skimmer potentials were used as follows: riddelliine $\mathrm{N}$-oxide $(\mathrm{m} / \mathrm{z}, 366)$ at $30 \mathrm{~V}$, riddelliine $(\mathrm{m} / \mathrm{z} 350)$ at $35 \mathrm{~V}$, monocrotaline internal standard $(\mathrm{m} / \mathrm{z}, 326)$ at $45 \mathrm{~V}$, and retronecine $(\mathrm{m} / \mathrm{z} 156)$ at $35 \mathrm{~V}$. Confirmatory fragment ions were monitored for all analytes at $m / z$ 136, 120, and 118 using $80 \mathrm{~V}$ and the proposed fragmentation reactions leading to these ions are shown in Scheme 2. Analyte quantification was achieved using the response ratio between the peak areas for internal standard and analyte. Figure 2 shows representative chromatograms for riddelliine and metabolites.

Animal handling procedures. For the toxicokinetics studies, ad libitumfed Fisher rats and $\mathrm{B} 6 \mathrm{C} 3 \mathrm{~F} 1$ mice of both sexes at 6-8 weeks of age were administered by oral gavage riddelliine $(4 \mathrm{mg} / \mathrm{mL}$ in $0.1 \mathrm{M}$ phosphate buffer) at a dose of $10 \mathrm{mg} / \mathrm{kg}$ body wt $(0.3 \mathrm{~mL} / 100 \mathrm{~g}$ body wt for rats, $0.1 \mathrm{~mL} / 10 \mathrm{~g}$ body wt for mice). After dosing, blood samples were collected at times selected from preliminary studies $(0.5-24 \mathrm{~h})$. In the rat studies, six sequential blood samples were collected into serum separation tubes from individuals through tail cuts (females, $n=5$; males, $n=3$ ). In the mouse studies, replicate animals were dosed and then blood was collected at euthanasia by cardiac puncture ( $n=6$ mice per time point for males and females). Serum was produced by centrifugation after clotting at room temperature and stored at $-20^{\circ} \mathrm{C}$ until analyzed. Serum concentrations of riddelliine and metabolite were determined using LC-ES/MS from aliquots of $10-100 \mu \mathrm{L}$ that were analyzed in duplicate for each determination.

Toxicokinetic analysis. Plots of serum analyte concentrations vs time were prepared for individual rats or groups of mice (see Figs. 3-6) and were analyzed using the model-independent spreadsheet approach of Ritschel (1986). Natural log-linear plots were fit to two phases attributed to distribution and elimination (see below). The first-order elimination rate constant $\left(k_{\beta}\right)$ was determined from the slope of the terminal phase of the In-linear blood concentration-time curve. A first-order distribution rate constant was determined 
A
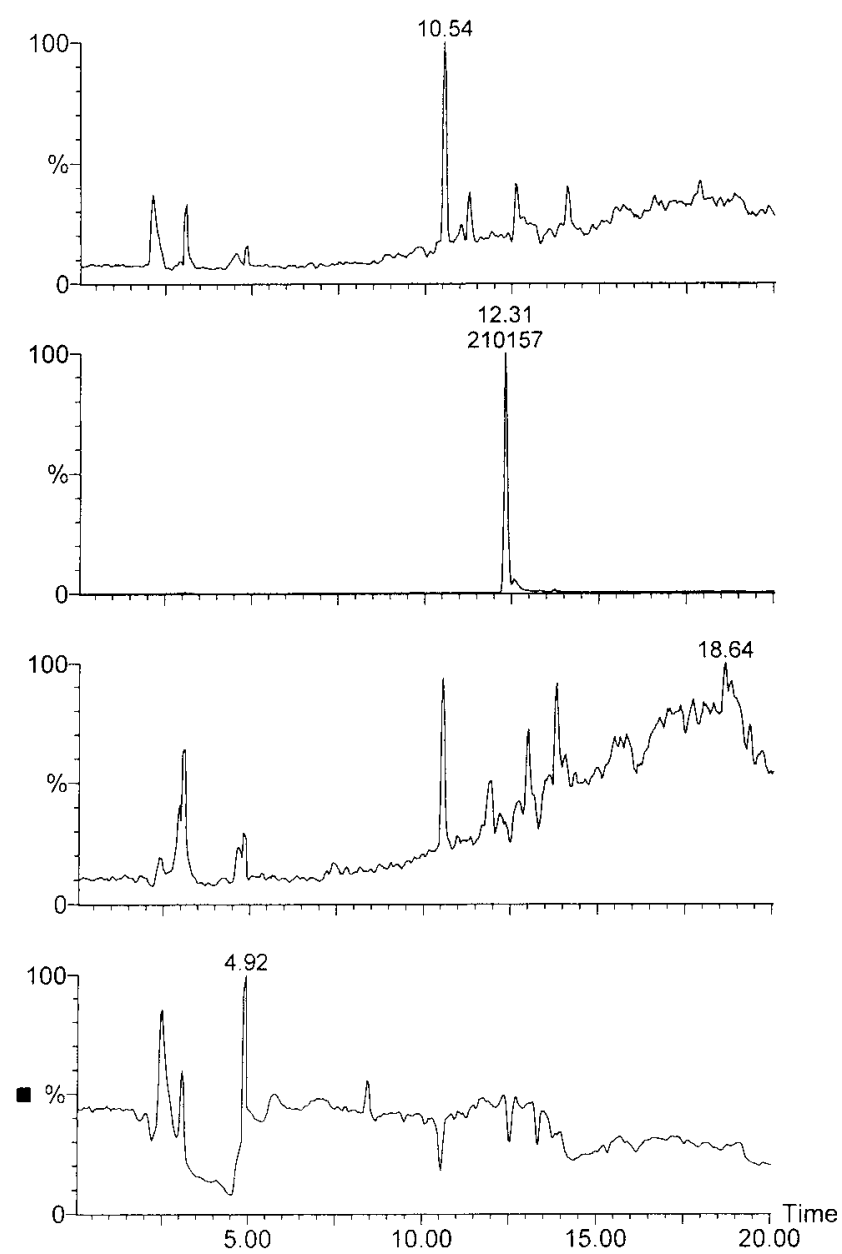

B
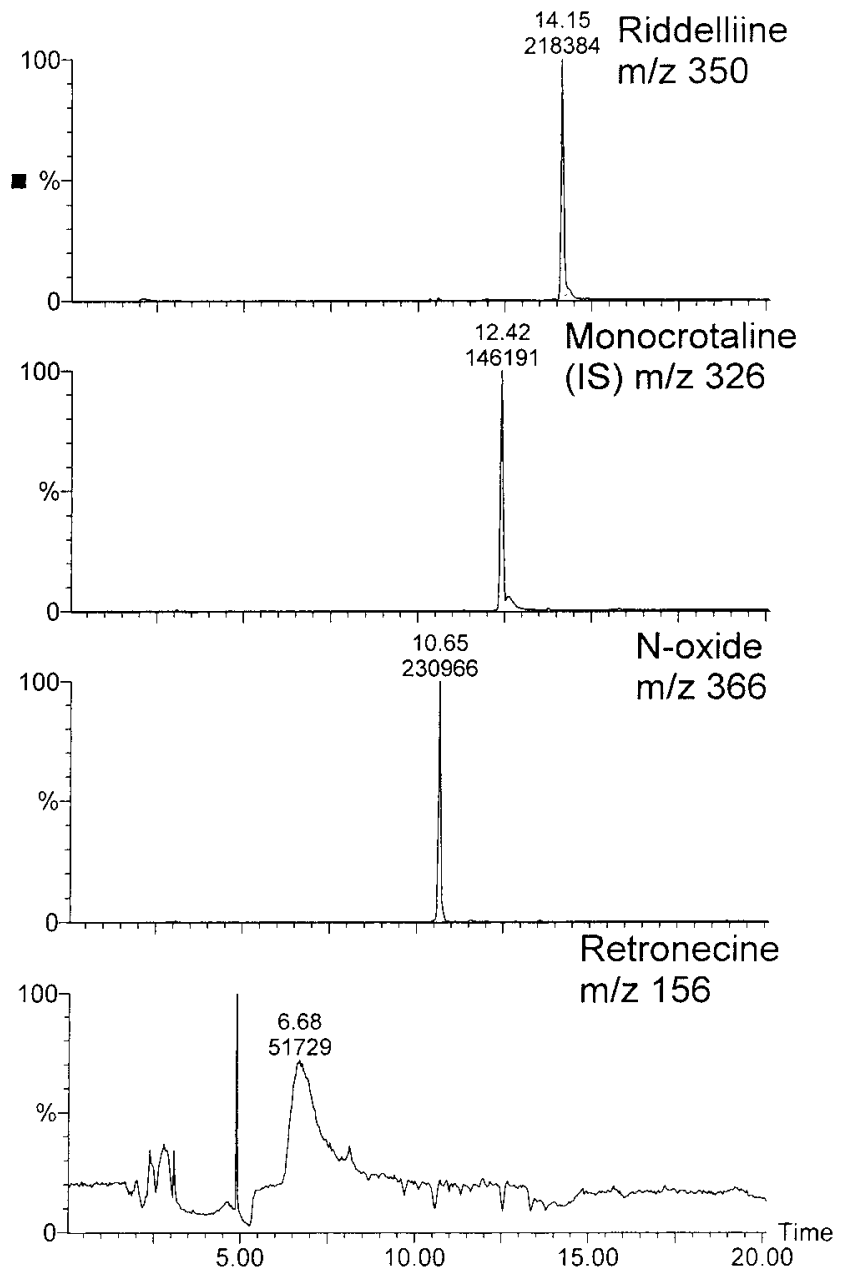

FIG . 2. LC-ES/MS chromatograms for riddelliine and metabolites in mouse serum $(\mathrm{A})$ The set of protonated molecule chromatograms for riddelliine $(\mathrm{m} / z$ 350), monocrotaline internal standard (IS, $\mathrm{m} / z, 326)$, riddelliine $N$-oxide $(\mathrm{m} / \mathrm{z}, 366$ ), and retronecine $(\mathrm{m} / \mathrm{z}$ 156) for untreated female mouse serum. (B) The analogous chromatograms for serum collected at $0.5 \mathrm{~h}$ from a female mouse gavaged with $10 \mathrm{mg} / \mathrm{kg}$ riddelliine.

from mean blood concentration-time plots after subtracting contributions from the terminal portion of the curve. Exposure to riddelliine and metabolites over time [area under the time-concentration curve from zero to infinity $\left(\mathrm{AUC}_{0-\infty}\right)$ ] was estimated for individual rats or groups of mice using the trapezoidal rule (Ritschel, 1986). The apparent volume of distribution for riddelliine $\left(V_{\mathrm{d}} / f\right)$ was calculated from dose/(AUC $\times k_{\beta}$ ). Insufficient amounts of the riddelliine metabolites were available for full toxicokinetic determinations, so measurements of $V_{\mathrm{d}} / f$ were not made. The two-tailed $t$ test was used to assess statistical significance $(p<0.05)$.

\section{RESULTS AND DISCUSSION}

\section{Method Validation}

It was necessary to use an internal standard to obtain accurate and precise quantification and stable labeled analogs were not available; for these reasons, monocrotaline was selected because of its close structural homology with riddelliine and similar chromaographic behavior. The recoveries of analytes from spiked serum were determined to be $102 \pm 10,79 \pm 7$, and $51 \pm 16 \%$ for riddelliine, the $N$-oxide, and retronecine, respectively (mean $\pm \mathrm{SD}, n=4-6$ ). The limits of quantification in $100 \mu \mathrm{L}$ serum, approximated by extrapolating to a signal/noise ratio of $10: 1$, for riddelliine and its $N$-oxide were $0.1 \mathrm{ng} / \mathrm{mL}$ and for retronecine $5 \mathrm{ng} / \mathrm{mL}$. Although the sensitivity for LC/MS detection of an aqueous retronecine standard was equivalent to the other analytes, the higher polarity of this compound led to a greater degree of interference from serum constituents. In addition, the presence of serum caused significant peak broadening over that seen for standards alone. The net result of these complications was lower overall sensitivity for retronecine in serum. The analytical method was validated for all three analytes at different concentrations by replicate analysis of spiked control rat serum $(100 \mu \mathrm{L})$ through the SPE and LC/MS procedures on different days. Table 1 shows the 


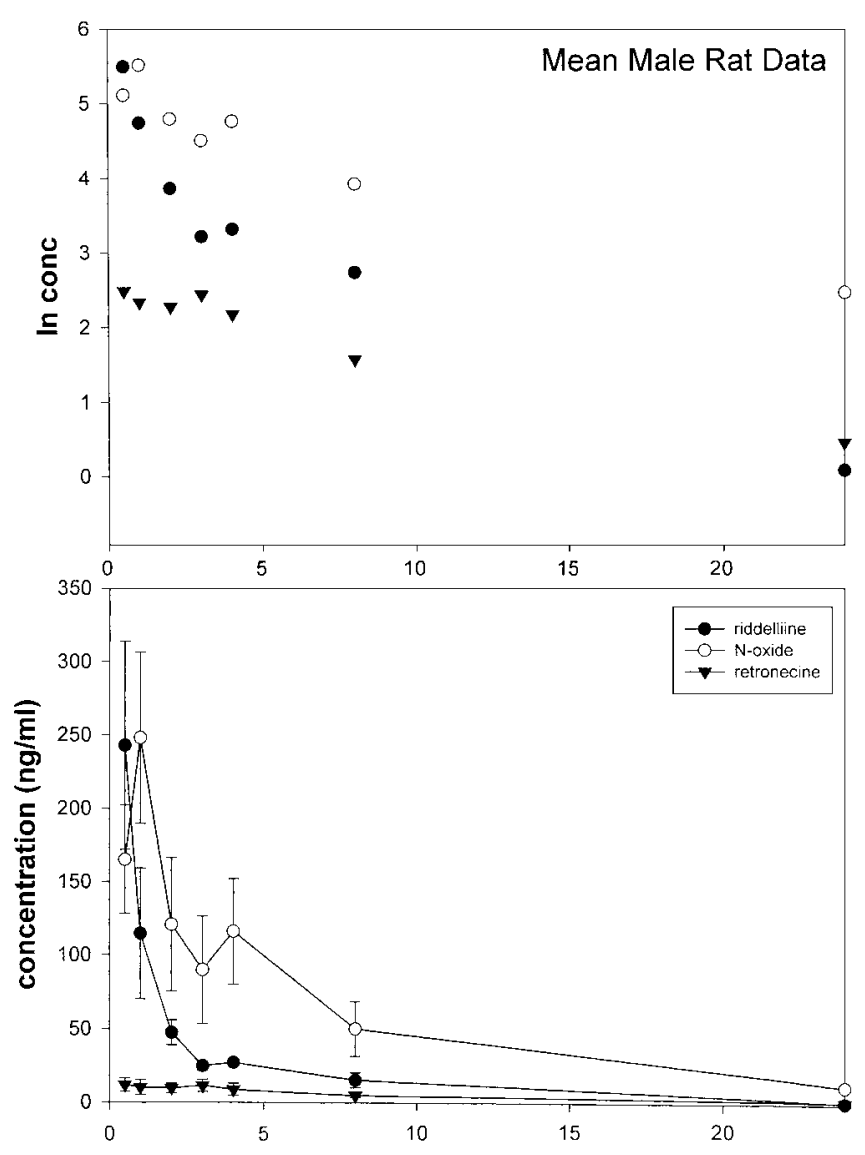

FIG. 3. Toxicokinetic plots for riddelliine and metabolites in male rat serum. The concentrations of analytes in serum were determined using the LC-ES/MS method for three individual rats and means \pm SD were plotted either as the concentration (bottom) or the natural logarithm of concentration (top) vs time following gavage dosing with $10 \mathrm{mg} / \mathrm{kg}$ of riddelliine.

results for the three analytes. The interday and intraday precision were acceptable for riddelliine, the $\mathrm{N}$-oxide, and retronecine, but the accuracy for retronecine analysis was affected by the above-mentioned serum interferences at low concentrations. It was not possible to determine serum concentrations of the important metabolite dehydroretronecine (DHR) because of its instability in aqueous solutions, especially in serum. Mass balance studies using riddelliic acid, the ester hydrolysis product formed concomitantly with DHR and retronecine, is a possible future avenue of investigation to better quantify activation vs detoxication pathways.

\section{Toxicokinetic Analysis}

The $10 \mathrm{mg} / \mathrm{kg}$ dose of riddelliine and the oral route of administration were selected based on results obtained from a 13 -week toxicity test in rats $(10 \mathrm{mg} / \mathrm{kg})$ and mice $(25 \mathrm{mg} / \mathrm{kg}$, Chan et al., 1994). Toxicokinetics were not determined for other doses. Analyte concentrations in serum were determined using the LC/MS method described above and model-indepen- dent analysis of the data was performed. Figures 3-6 show the plots of mean serum concentrations of riddelliine and metabolites for rats and mice. The mean rat data shown are representative of the individual data. These plots show that riddelliine was completely absorbed from the gavage dose within 30 min in all rats and mice. The plots also show rapid and extensive conversion of riddelliine to the $\mathrm{N}$-oxide, with the exception that female rats produced lower serum concentrations of the $\mathrm{N}$-oxide. All rodents produced small amounts of retronecine. Table 2 shows the compilation of toxicokinetic parameters for all compounds in rats and mice. Note that values shown with SDs were determined from plots using individual rat serum concentration data and those without SDs were determined from plots using averages from six individual mouse serum samples at each time point. Distribution and elimination rate processes were observed except for retronecine for which the ln-concentration vs time plots were consistent with a single rate process of elimination.

The elimination half-times increased in the following order: riddelliine $<$ retronecine $<N$-oxide consistent with metabo-

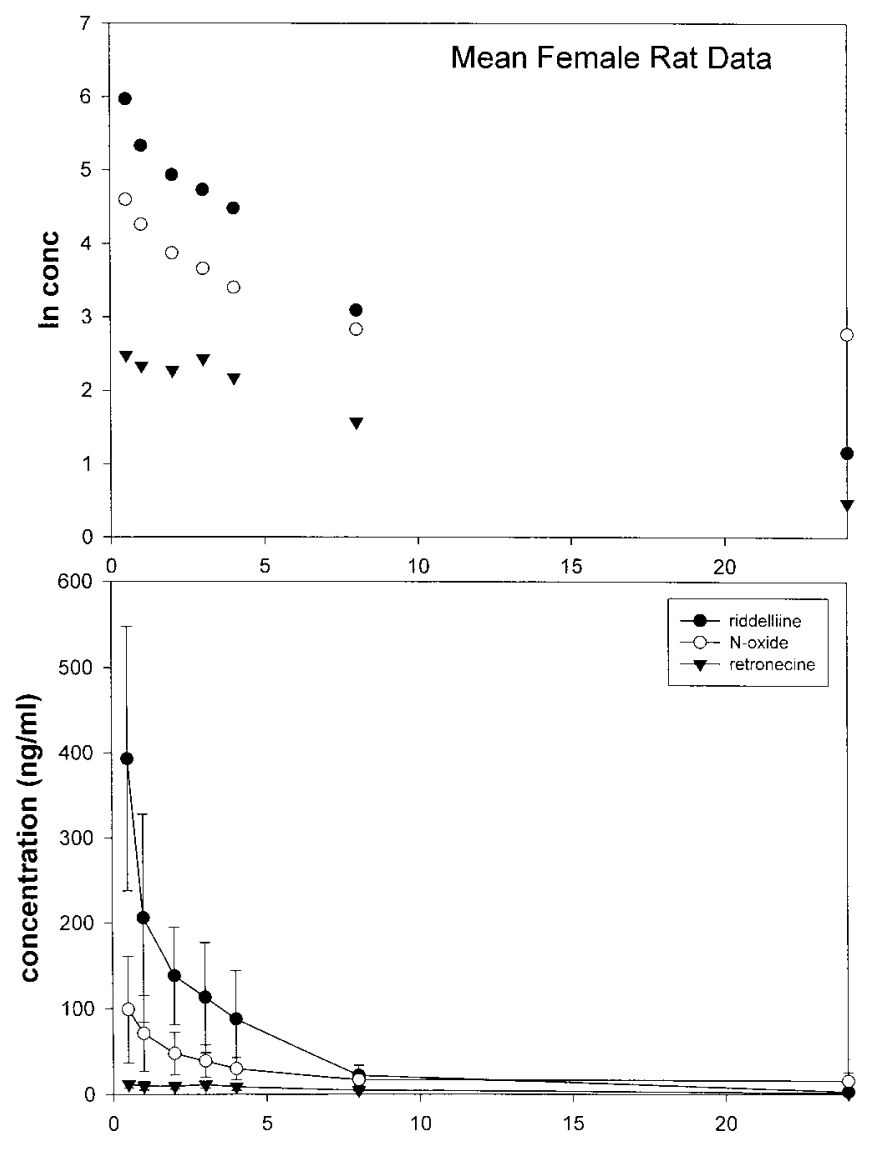

FIG . 4. Toxicokinetic plots for riddelliine and metabolites in female rat serum. The concentrations of analytes in serum were determined using the LC-ES/MS method for five individual rats and means were plotted either as the concentration (bottom) or the natural logarithm of concentration (top) vs time following gavage dosing with $10 \mathrm{mg} / \mathrm{kg}$ of riddelliine. 


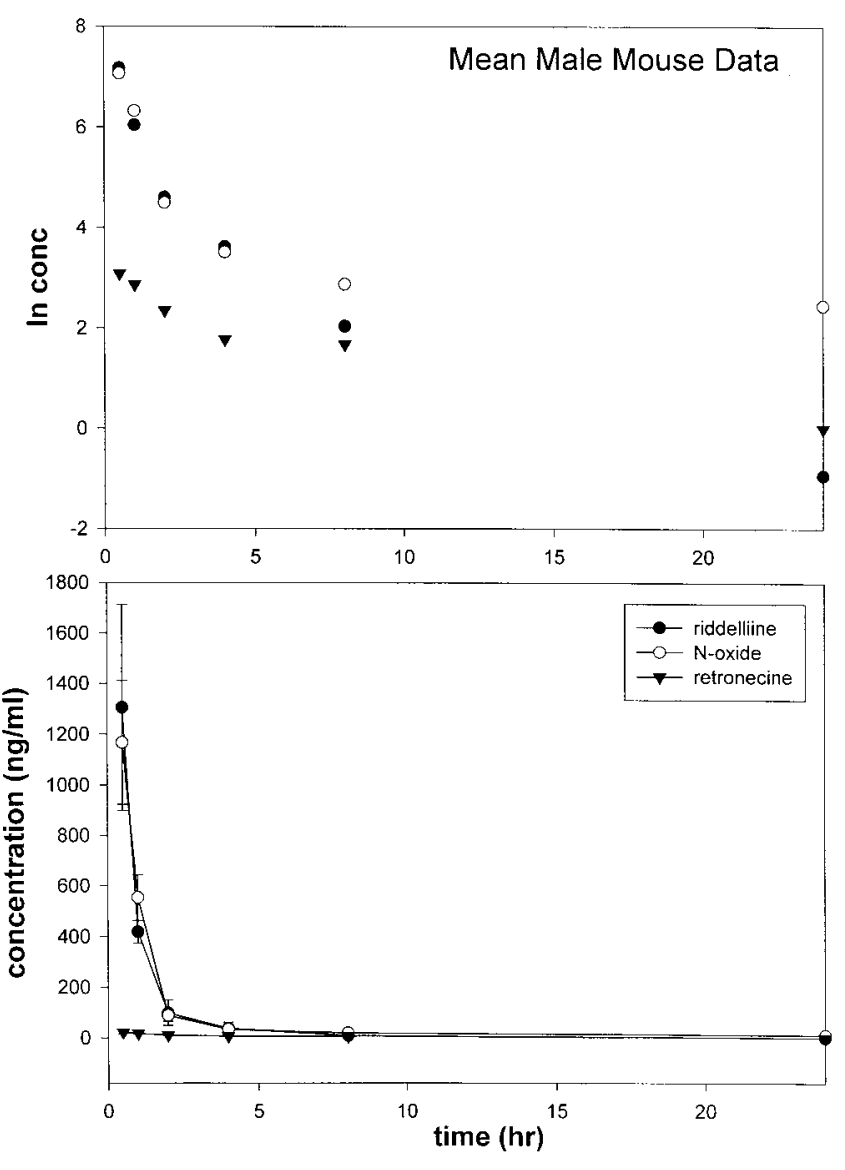

FIG . 5. Toxicokinetic plots for riddelliine and metabolites in male mouse serum. The concentrations of analytes in serum were determined using the LC-ES/MS method for six mice per time point and means \pm SD were plotted either as the concentration (bottom) or the natural logarithm of concentration (top) vs time following gavage dosing with $10 \mathrm{mg} / \mathrm{kg}$ of riddelliine.

lism of parent compound. Internal exposures $\left(\mathrm{AUC}_{0-\infty}\right)$ increased in the order: retronecine $<$ riddelliine $<N$-oxide, with male rats as the exception. The small body weight-normalized volume of distribution $\left(V_{\mathrm{d}} / f\right)$ for riddelliine is consistent with limited tissue distribution. Half-times for the apparent distribution processes of riddelliine and the $\mathrm{N}$-oxide were comparable for mice and rats. Taken together, these findings are consistent with extensive metabolism of riddelliine and subsequent elimination of the polar metabolites.

The half-time for riddelliine elimination from male and female rats was similar. The half-time for riddelliine elimination from male and female mice was similar, although faster than that observed in rats. The half-time for distribution of riddelliine was comparable for all rats and mice. The internal exposure $\left(\mathrm{AUC}_{0-\infty}\right)$ for the $\mathrm{N}$-oxide was greater than that for riddelliine in male rats, but this relationship was reversed in female rats. This observation is consistent with related studies using isolated liver microsomes in which male rats produced approximately five-fold more riddelliine $N$-oxide and dehydroretronecine than females (data not shown). This parallel sug- gests that oxidative hepatic metabolism of riddelliine results in a diminished internal exposure to the parent compound and increased exposure to the $\mathrm{N}$-oxide and dehydroretronecine metabolites. This conclusion is supported by the previous finding of apparent greater sensitivity of male rats, relative to females, to the toxic effects of riddelliine in a 13-week exposure study (Chan et al., 1994). The greater $V_{\mathrm{d}} / f$ for riddelliine in male rats relative to females, which correlates with increased distribution of riddelliine into tissues, is also consistent with the increased toxicity seen in male rats. The $V_{\mathrm{d}} / f$ for riddelliine, and the AUC for either riddelliine or the $\mathrm{N}$-oxide, were similar in male and female mice; however, the AUC for $\mathrm{N}$-oxide was greater than that for riddelliine in both mouse groups. Although mouse liver microsomal incubations with riddelliine were not performed, the AUC comparison predicts that similar rates of $\mathrm{N}$-oxide formation would occur in both males and females. No species or sex differences in the half-time of elimination and AUCs for retronecine were observed.
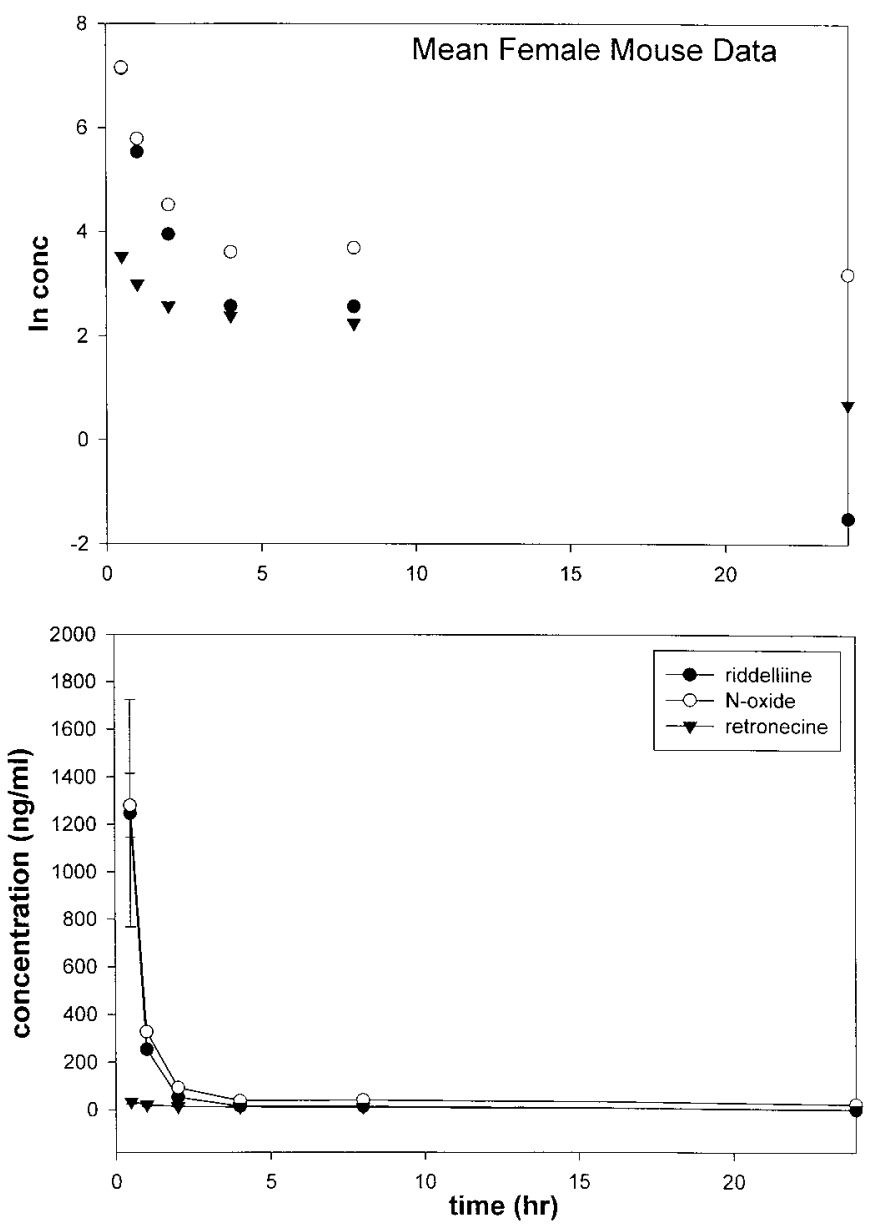

FIG. 6. Toxicokinetic plots for riddelliine and metabolites in female mouse serum. The concentrations of analytes in serum were determined using the LC-ES/MS method for six mice per time point and means \pm SD were plotted either as the concentration (bottom) or the natural logarithm of concentration (top) vs time following gavage dosing with $10 \mathrm{mg} / \mathrm{kg}$ of riddelliine. 
TABLE 1

Method Validation Summary

\begin{tabular}{|c|c|c|c|c|}
\hline & $\begin{array}{l}\text { Day } 1 \text { precision } \\
\text { (RSD) }\end{array}$ & $\begin{array}{c}\text { Day } 1 \text { accuracy } \\
\text { (\% of amount added) }\end{array}$ & $\begin{array}{l}\text { Day } 2 \text { precision } \\
\text { (RSD) }\end{array}$ & $\begin{array}{c}\text { Day } 2 \text { accuracy } \\
\text { (\% added })\end{array}$ \\
\hline \multicolumn{5}{|c|}{ [Riddelliine] (ng/mL) } \\
\hline 2 & $1.9 \pm 0.1(7.2)$ & 95 & $1.8 \pm 0.1(3.5)$ & 90 \\
\hline 5 & $6.0 \pm 0.6(10.6)$ & 120 & $5.0 \pm 0.1(1.5)$ & 100 \\
\hline 40 & $39.6 \pm 2.5(6.3)$ & 99 & $43.1 \pm 4.1(9.4)$ & 108 \\
\hline \multicolumn{5}{|c|}{ [Riddelliine $N$-oxide] (ng/mL) } \\
\hline 2 & $2.0 \pm 0.3(15.4)$ & 100 & $1.7 \pm 0.1(6.4)$ & 85 \\
\hline 5 & $5.0 \pm 0.6(12)$ & 100 & $5.2 \pm 0.6(11.4)$ & 104 \\
\hline 40 & $39.0 \pm 0.7(1.9)$ & 98 & $44.9 \pm 3.9(8.7)$ & 90 \\
\hline \multicolumn{5}{|c|}{ [Retronecine] (ng/mL) } \\
\hline 5 & $7.0 \pm 0.7(10)$ & 140 & $5.7 \pm 0.3(5.3)$ & 114 \\
\hline 40 & $33.0 \pm 3.3(9.9)$ & 83 & $31.9 \pm 3.0(9.4)$ & 80 \\
\hline
\end{tabular}

Note. Serum from untreated rats was spiked with various concentrations of riddelliine and metabolites and analyzed on two separate days. The values shown are means $\pm \mathrm{SD}$ with the relative standard deviation $(\mathrm{RSD}=100 \times \mathrm{SD} /$ mean) expressed as a percentage shown in parentheses.

The slower elimination of riddelliine $N$-oxide from serum, relative to riddelliine, increases the extent to which additional metabolism of the $\mathrm{N}$-oxide could occur, including microsomal reduction back to the parent amine. This is a possible mechanism for extending exposures to parent pyrrolizidine in the tissues and enhancing toxicity through subsequent metabolism to dehydroretronecine. This notion is consistent with our findings that administration of riddelliine $\mathrm{N}$-oxide to rats led to formation of the same DNA adducts in liver as seen from either the parent compound or the activated metabolite dehydroretronecine (Fu et al., 2001). This conclusion is important because it has been widely presumed that pyrrolizidine $\mathrm{N}$-oxides are nontoxic metabolites. On the other hand, the hydrolysis of riddelliine to form retronecine, albeit in small quantities, is a detoxification process because DNA adduct formation was not observed following microsomal metabolism of retronecine ( $\mathrm{Fu}$ et al., 2001). Scheme 1 summarizes these proposed metabolic transformations of riddelliine.

A previous study of pyrrolizidine toxicokinetics in rats was performed using radiolabeled monocrotaline (Estep et al., $1991)$. This study showed rapid urinary $(>80 \%)$ and biliary $(>10 \%)$ excretion of total monocrotaline-derived radioactivity. Approximately $60 \%$ of the radioactivity in urine was due to the parent compound and $<4 \%$ was from the $N$-oxide. Little parent compound or $\mathrm{N}$-oxide was observed in bile, although products associated with metabolic conversion to reactive pyrroles (pos-

TABLE 2

Compilation of Toxicokinetic D eterminations for R iddelliine and Metabolites

\begin{tabular}{|c|c|c|c|c|}
\hline & $\begin{array}{c}\text { Elimination } t_{1 / 2} \\
\text { (h) }\end{array}$ & $\begin{array}{l}\text { Distribution } t_{1 / 2} \\
\text { (h) }\end{array}$ & $\begin{array}{c}\mathrm{AUC}_{0-\infty} \\
(\mathrm{ng} \mathrm{h} / \mathrm{mL})\end{array}$ & $\begin{array}{c}V_{\mathrm{d}} / f \\
(\mathrm{~L} / \mathrm{kg} \text { body wt })\end{array}$ \\
\hline \multicolumn{5}{|l|}{ Riddelliine } \\
\hline Rat male & $4.2 \pm 0.3$ & 0.35 & $516 \pm 80^{*}$ & $120 \pm 16^{*}$ \\
\hline Rat female & $4.2 \pm 1.0$ & 0.55 & $1267 \pm 395$ & $53 \pm 29$ \\
\hline Mouse male & 3.2 & 0.34 & 1307 & 35 \\
\hline Mouse female & 3.0 & 0.24 & 1064 & 41 \\
\hline \multicolumn{5}{|l|}{ Riddelliine $N$-oxide } \\
\hline Rat male & $7.0 \pm 1.3$ & 0.55 & $1494 \pm 367 *$ & \\
\hline Rat female & $11.9 \pm 7.2$ & 0.37 & $714 \pm 405$ & \\
\hline Mouse male & 15.4 & 0.35 & 1753 & \\
\hline Mouse female & 28.9 & 0.33 & 2746 & \\
\hline \multicolumn{5}{|l|}{ Retronecine } \\
\hline Rat male & $8.2 \pm 1.0$ & NA & $88 \pm 24$ & \\
\hline Rat female & $6.7 \pm 1.8$ & NA & $135 \pm 36$ & \\
\hline Mouse male & 6.9 & NA & 128 & \\
\hline Mouse female & 8.1 & NA & 217 & \\
\hline
\end{tabular}

Note. NA, not applicable.

* Significant sex difference $(p<0.05)$. 
sibly dehydroretronecine) were observed in bile. These results are also consistent with the notion that further metabolism of the $N$-oxide is required prior to excretion.

Preliminary results from the 2-year carcinogenicity bioassay of riddelliine in male and female rats and mice showed that all species/sex combinations were susceptible to hemangiosarcomas and hepatocellular tumors, with the exception of female mice. Although the toxicokinetic data presented here are in agreement with the gross toxicity results in male and female rats (Chan et al., 1994), it is clear that additional factors (e.g., dehydroretronecine formation) are responsible for the species/ sex differences seen in tumorigenic susceptibility.

\section{ACKN OW LED GMENTS}

This research was supported in part by Interagency Agreement 224-93-0001 between the National Center for Toxicological Research/Food and Drug Administration and the National Institute for Environmental Health Sciences/ National Toxicology Program. L.W. acknowledges support of a fellowship from the Oak Ridge Institute for Science and Education, administered through an interagency agreement between the U.S. Department of Energy and the U.S. Food and Drug Administration.

\section{REFERENCES}

Betz, J. M., Eppley, R. M., Taylor, W. C., and Andrzejewski, D. (1994). Determination of pyrrolizidine alkaloids in commercial comfrey products (Symphytum sp.). J. Pharm. Sci. 83, 649-653.

Chan, P. C., Mahler, J., Bucher, J. R., Travlos, G. S., and Reid, J. B. (1994). Toxicity and carcinogenicity of riddelliine following 13 weeks of treatment to rats and mice. Toxicon 32, 891-908.

Estep, J. A., Lame, M. W., Morin, D., Jones, A. D., Wilson, D. W., and Segall,
H. J. (1991). $\left[{ }^{14} \mathrm{C}\right]$ Monocrotaline kinetics and metabolism in the rat. Drug Metab. Disp. 19, 135-139.

Fu, P. P., Chou, M. W., Xia, Q., Yang, Y. C., Yan, J., Doerge, D. R., and Chan, P. C. (2001). Genotoxic pyrrolizidine alkaloids and pyrrolizidine alkaloid $\mathrm{N}$-oxides: Mechanisms leading to DNA adduct formation and tumorigenicity. Environ. Carcinogen. Ecotoxicol. Rev. 19, 353-385.

Hirono, I., Mori, H., and Culvenor, C. C. J. (1976). Carcinogenic activity of coltsfoot, Tussilago farfara L. Gann 67, 125-129.

Huxtable, R. J. (1980). Herbal teas and toxins: Novel aspects of pyrrolizidine poisoning in the United States. Perspect. Biol. Med. 24, 1-14.

International Agency for Research on Cancer (1976). Pyrrolizidine Alkaloids. In IARC Monographs on the Evaluation of Carcinogenic Risk of Chemicals to Man: Some Naturally Occurring Substance, Vol. 10, pp. 265-342. International Agency for Research on Cancer, Lyon.

Mattocks, A. R. (1986). Chemistry and Toxicology of Pyrrolizidine Alkaloids. Academic Press, New York.

National Toxicology Program (2001). Toxicology and Carcinogenesis Studies of Riddelliine (CAS No. 23246-96-0) in F344/N Rats and B6C3F1 Mice (Gavage Studies). NTP Technical Report 508, NIH Publication 01-4442, U.S. Dept. Health and Human Services, Public Health Service, National Institutes of Health, Bethesda, MD.

Ritschel, W. A. (1986). AUC-RPP: BASIC computer program for compartment model independent pharmacokinetic analysis. Methods Find. Exp. Clin. Pharmacol. 8, 633-640.

Roeder, E. (1995). Medicinal plants in Europe containing pyrrolizidine alkaloids. Pharmazie 50, 83-98.

Yang, Y. C., Yan, J., Churchwell, M. I., Beger, R., Chan, P. C., Doerge, D. R., Fu, P. P., and Chou, M. W. (2001a). Development of a ${ }^{32}$ P-postlabeling method for detection of dehydroretronescine-derived DNA adducts in vivo and in vitro. Chem. Res. Toxicol. 14, 91-100.

Yang, Y. C., Yan, J., Doerge, D. R., Chan, P. C., Fu, P. P., and Chou, M. W. (2001b). Metabolic activation of the tumorigenic pyrrolizidine alkaloid, riddelliine, leading to DNA adduct formation in vivo. Chem. Res. Toxicol. 14, 101-109. 